\title{
Preparation of ZnO-GO nanocomposites and their properties
}

\author{
Caifeng Jin ${ }^{1}$, Yuqing Cheng ${ }^{1}$, Wenbin $\mathrm{Liu}^{2}$, and Yuguang $\mathrm{Lv}^{1, *}$ \\ ${ }^{1}$ College of Pharmacy, Jiamusi University, Jiamusi, China \\ ${ }^{2}$ School of materials science and Engineering, Jiamusi University, Jiamusi, China
}

\begin{abstract}
ZnO}$ nanoparticles were prepared by hydrothermal method. The modified Hummer process was then used to prepare go. The $\mathrm{ZnO}-\mathrm{GO}$ com posites were then mixed to produce different ratios of $\mathrm{ZnO}-\mathrm{GO}$, characteris ed and their photocatalytic properties were investigated using rhodamine $\mathrm{B}$ as the target degradation species. The results showed that the degradation $\mathrm{r}$ ates of rhodamine $\mathrm{B}$ were $41.96 \%$ and $84.42 \%$ for the nano- $\mathrm{ZnO}$ and $\mathrm{ZnO}-$ GO 7\% composites, respectively, when illuminated with xenon light for 36 $0 \mathrm{~min}$. The improved photocatalytic activity of composite materials is prim arily due to the introduction of appropriate amount of GO, which could ra pidly capture and transfer to the photogenerated electrons, and could load more $\mathrm{ZnO}$ and prevent the agglomeration of $\mathrm{ZnO}$ particles, increasing the $\mathrm{s}$ pecific surface area of $\mathrm{ZnO}$ and its activation point, as well as increasing th e adsorption of pollutants on the $\mathrm{ZnO}$ surface and improving the photocatal ytic efficiency.
\end{abstract}

Keywords: Namo-ZnO; Graphene oxide; Photocatalysis.

\section{Introduction}

As society develops and environmental problems such as waste pollution become increasingly prominent, a sewage treatment method is urgently needed. Semiconductor oxide photocatalytic oxidation technology is a relatively new type of technology for treating water pollution. As a photocatalyst, semiconductor oxides include $\mathrm{TiO}_{2}, \mathrm{ZnO}, \mathrm{WO}_{3}$, $\mathrm{SnO}_{2}$, etc. Among them, the redox capacity of titanium dioxide is very strong, chemically stable, non-toxic, and does not cause secondary pollution.Ban bandwidth, ban bandwidth zinc oxide closer to titanium dioxide, and zinc oxide synthesis with a low cost, is a metal oxide semiconductor material that can replace titanium dioxide for the effective degradation of the organic dye contaminant ${ }^{[1-2]}$. Nanoparticles will exhibit many excellent properties compared to normal $\mathrm{ZnO}$, such as non-toxicity, piezoelectricity, fluorescence, and the ability to absorb and scatter ultraviolet light. The main ways to improve the photocatalytic activity of $\mathrm{ZnO}$ nanomaterials are: firstly, to control the $\mathrm{ZnO}$ growth structure; and secondly, to carry out surface modifications. At present, the problems encountered in the process of overcoming composite graphene are often solved by using surface modification, i.e. replacing graphene with graphene oxide. In this study, $\mathrm{ZnO}$ nanoparticles were firstly prepared by hydrothermal method; then graphene oxide (GO) was prepared by modified

*Corresponding author: yuguanglv@163.com 
Hummers method; the two were then mixed to prepare $\mathrm{ZnO}-\mathrm{GO}$ composites in different ratios, and their photocatalytic properties were investigated using rhodamine $\mathrm{B}$ as the target degradant $^{[3]}$.

\section{Catalyst material preparation}

\subsection{Preparation of ZnO-GO composites}

The $\mathrm{ZnO}$ dispersion was obtained by adding $50 \mathrm{ml}$ of anhydrous ethanol to a beaker and sonicating for $2 \mathrm{~h}$. The graphene oxide ethanol suspension was added to the $\mathrm{ZnO}$ dispersion ( $50 \mathrm{ml}$ of anhydrous ethanol was added to $\mathrm{GO}$ and sonicated for $2 \mathrm{~h}$ to obtain an ethanol suspension of graphene oxide) and stirred at elevated temperature for $24 \mathrm{~h}$. The resulting product was washed by centrifugation and anhydrous ethanol. The product was then redispersed in $10 \mathrm{~mL}$ of anhydrous ethanol. Vacuum drying for $24 \mathrm{~h}$ to obtain $\mathrm{ZnO}-\mathrm{GO}$ nanoparticles (ZnO-GO1\%, ZnO-GO3\%, ZnO-GO5\%, ZnO-GO7\%, ZnO-GO10\% were produced respectively)

\section{Results and Discussion}

\subsection{Structural and morphological characterisation}

Figure 1(a) shows the XRD pattern of the morphology of the $\mathrm{ZnO}$ nanoparticles prepared by the hydrothermal method. All diffraction peaks match the standard data for the fibrillated $\mathrm{ZnO}$ structure of the $\mathrm{ZnO}$ hexagonal crystal system (JPCDS 36-1451). The sharp shape of the diffraction peaks in the figure indicates that the obtained $\mathrm{ZnO}$ crystals are well crystalline. The XRD patterns of both are highly compatible and the absence of their characteristic diffraction peaks in the XRD patterns indicates that the synthesised samples are both of high purity and no impurities are produced. No diffraction peaks of graphene were observed in the XRD patterns due to the small proportion of graphene oxide in the catalyst.The XRD patterns of the composites (c) show that the doping of different amounts of graphene oxide resulted in lower intensity and wider peak shape of $\mathrm{ZnO}$ compared to pure $\mathrm{ZnO}$, and a decrease in crystallinity of the composite samples. The effect on the crystallinity of $\mathrm{ZnO}$ gradually increases as the amount of graphene oxide doping increases $^{[5]}$. In addition, the SEM and TEM photographs given in Figure 2 show that the $\mathrm{ZnO}$ particles are attached to the surface layer of graphene oxide, and the graphene oxide doping obviously prevents the agglomeration of $\mathrm{ZnO}$ nanoparticles.

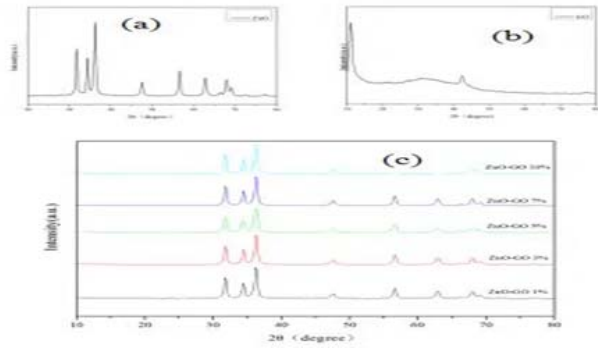

Fig. 1. XRD spectra of $\mathrm{ZnO}$ (a), GO (b), $\mathrm{ZnO}-\mathrm{GO}$ composites (c).

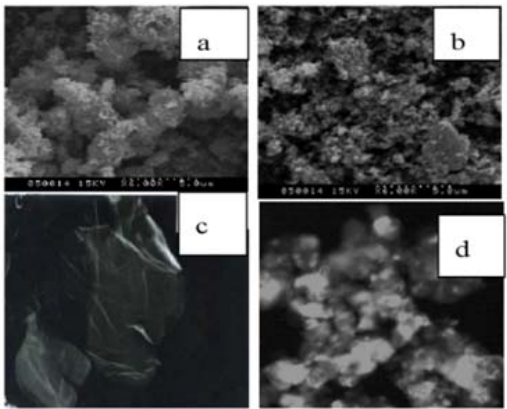

Fig. 2. Scanning electron micro-graphs of $\mathrm{ZnO}$ nanoparticles (a) and $\mathrm{ZnO}-\mathrm{GO} 1 \%(\mathrm{~b}), \mathrm{GO}(\mathrm{c}), \mathrm{ZnO}-$ $\mathrm{GO} 1 \%$ (d) transmission electron micrographs. 


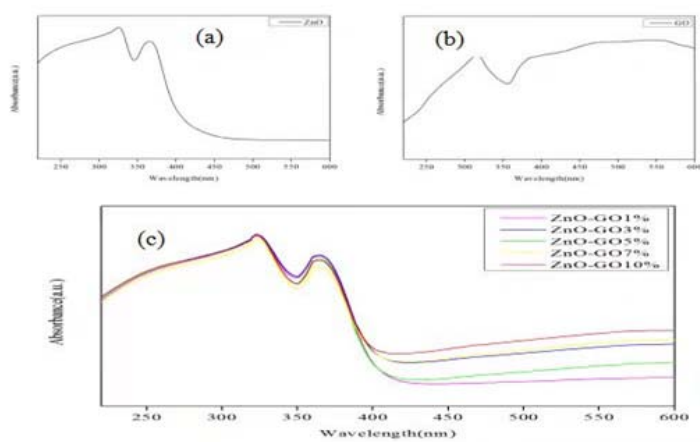

Fig. 3. UV-Vis spectra of $\mathrm{ZnO}(\mathrm{A})$, GO (B), and $\mathrm{ZnO}-\mathrm{GO}$ composites with different concentrations (C).

Figure 3 shows the UV-Vis absorption spectra of different amounts of graphene oxide doped $\mathrm{ZnO}$, from which it can be seen that all samples have strong absorption at $385 \mathrm{~nm}$, corresponding to the intrinsic band width of $\mathrm{ZnO}$ nanoparticles. The absorption intensity of the graphene oxide doped photocatalysts was significantly enhanced in the visible range, indicating that the addition of graphene oxide altered the light absorption properties of $\mathrm{ZnO}$.

\section{Degradation of rhodamine B by ZnO-GO composite photocatalyst}

\subsection{Photocatalytic methods}

$50 \mathrm{mg}$ of photocatalyst and $100 \mathrm{~mL}$ of rhodamine B solution at a concentration of $10 \mathrm{mg} / \mathrm{L}$ were weighed separately in a $100 \mathrm{~mL}$ double beaker. The solution was magnetically stirred in the dark for a certain period. A xenon light source and condensation unit were then switched on for visible light-catalyzed degradation. Approximately $5 \mathrm{~mL}$ of the reaction solution is extracted at regular intervals, the catalyst is removed by centrifugation and the supernatant is measured by UV-Vis spectrophotometer at a maximum absorption wavelength of $554 \mathrm{~nm}$ to determine the residual concentration of the catalytic degradation and to calculate the degradation rate $\eta^{\left[{ }^{[6]}\right.}$. The formula was calculated as:

$$
\eta \%=1-C_{t} / C_{0} \times 100 \%=1-A_{t} / A_{0} \times 100 \%
$$

t-reaction time $(\mathrm{min})$.

$\mathrm{C}_{\mathrm{t}}$-concentration of pollutant at time $\mathrm{t}(\mathrm{mg} / \mathrm{L})$.

$\mathrm{C}_{0}$-equilibrium concentration of pollutant after dark adsorption-desorption equilibrium $(\mathrm{mg} / \mathrm{L})$.

$A_{t}$ - the absorbance of the contaminant at time $t$.

$\mathrm{A}_{0}$-absorbance of the pollutant after dark adsorption-desorption equilibrium.

\subsection{Photocatalytic properties}




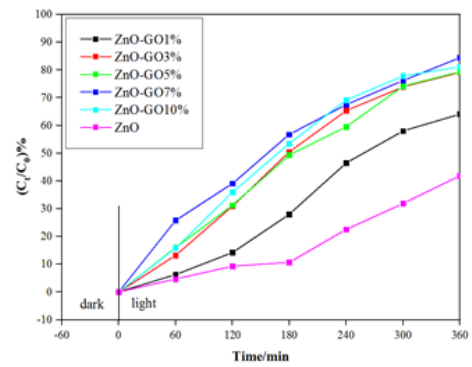

Fig. 4. Effect of different GO concentrations on the photocatalytic performance of the catalyst.

The photocatalytic activity of rhodamine B was tested by desorption of the sample under UV light. The reaction was preceded by adsorption in a dark box for $30 \mathrm{~min}$ and after reaching adsorption equilibrium, the UV light source was switched on. Figure 4 shows the photocatalytic activity of the different samples ${ }^{[7]}$. It can be seen that the $\mathrm{ZnO}$ nanopowder has a certain degradation effect on rhodamine B, with a degradation rate of $41.96 \%$ at $360 \mathrm{~min}$ of light exposure. From the photodegradation curves of the $\mathrm{ZnO}-\mathrm{GO}$ composites for rhodamine $\mathrm{B}$, more $\mathrm{GO}$ content does not necessarily mean that the photocatalytic performance of the composites is good, probably because too much GO content in the composites will instead affect the absorption of UV light by $\mathrm{ZnO}$, thus affecting the photoexcitation and catalytic activity of the $\mathrm{ZnO}$ particles. The proper introduction of GO can make the composite adsorb organic dyes well and can promote the effective separation of $\mathrm{ZnO}$ photogenerated carriers and reduce the composite rate. This shows that the ratio of $\mathrm{ZnO}$ and $\mathrm{GO}$ in the composite has a very strong influence on photocatalysis. Among them, $\mathrm{ZnO}-\mathrm{GO} 7 \%$ has the best photocatalytic performance, with a degradation rate of $84.42 \%$ at 360 min of light.

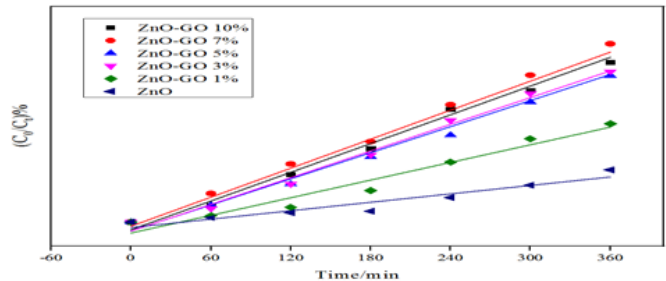

Fig. 5. $\ln (\mathrm{C} 0 / \mathrm{Ct})-\mathrm{t}$ linear fit curves for catalyst samples with different $\mathrm{GO}$ concentrations.

Table 1. Linear correlation coefficients between reaction rate constants and regression equations for different catalysts.

\begin{tabular}{|c|c|c|c|}
\hline Sample name & Regression equation & $\mathrm{K}$ & $\mathrm{R}^{2}$ \\
\hline $\mathrm{ZnO}$ & $y=0.0995 x-0.1077$ & 0.00147 & 0.928 \\
\hline $\mathrm{ZnO}-\mathrm{GO} 1 \%$ & $y=0.2975 x-0.1769$ & 0.00307 & 0.9951 \\
\hline $\mathrm{ZnO}-\mathrm{GO} 3 \%$ & $y=0.2906 x-0.1733$ & 0.00464 & 0.9887 \\
\hline $\mathrm{ZnO}-\mathrm{GO} 5 \%$ & $y=0.2069 x-0.2134$ & 0.00500 & 0.9791 \\
\hline $\mathrm{ZnO}-\mathrm{GO} 7 \%$ & $y=0.3114 x-0.0813$ & 0.00504 & 0.9909 \\
\hline ZnO-GO10\% & $y=0.316 x-0.1506$ & 0.00453 & 0.9895 \\
\hline
\end{tabular}

Figure 5 shows the linear fitting curve of $\ln \left(\mathrm{C}_{0} / \mathrm{C}_{\mathrm{t}}\right)$-t for $\mathrm{ZnO}-\mathrm{GO} 7 \%$. It can be seen that $\ln \left(\mathrm{C}_{0} / \mathrm{C}_{\mathrm{t}}\right)$-t shows a good linear correlation, indicating that the photocatalytic degradation of 
rhodamine $\mathrm{B}$ is an apparent primary reaction. The rate constants and linear correlation coefficients of the regression equations are shown in Table 1, which shows that $\mathrm{ZnO}-\mathrm{GO} 7 \%$ had the best catalytic effect with the highest rate constant of 0.00504 . The kinetic fitting results are consistent with the degradation efficiency results in Figure 4. The linear correlation coefficients of the regression equations were all greater than or close to 0.99 , indicating a good regression effect.

\section{Conclusion}

Zno nanoparticles were obtained by simple hydrothermal method, and go and GO were obtained by modified Hummer method, and $\mathrm{ZnO}-\mathrm{GO}$ composites with different GO concentrations were prepared by mixing $\mathrm{ZnO}$ nanoparticles with GO. Here we study the efficiency of rhodamine B as a pollutant for photocatalytic degradation by using a xenon lamp greater than $400 \mathrm{~nm}$ as the unique light source.The experimental results showed that $\mathrm{ZnO}-\mathrm{GO} 7 \%$ had the best catalytic effect with $84.42 \%$ degradation rate when the light time was $360 \mathrm{~min}$. The composite material is simple to prepare and has certain practical application value.

\section{References}

1. Ling Sun. Structure and synthesis of graphene oxide[J]. Chinese Journal of Chemical Engineering. 2019(10)

2. ANDrew T.Smith,Anna Marie La Chance,Songshan Zeng, Bin Liu,Luyi Sun. Synthesis, properties, and applications of graphene oxide/reduced graphene oxide and their nanocomposites[J]. Nano Materials Science. 2019(01)

3. Xiao-Jun Shen, Shu Yang,Jian-Xiang Shen,Jun-Li Ma,Yin-Qiu Wu,Xiao-Ling Zeng,Shao-Yun Fu.Improved mechanical and antibacterial properties of silvergraphene oxide hybrid/polylactid acid composites by in-situ polymerization[J]. Industrial Crops \& Products . 2019

4. Cheng Zhang,Zhigao Wang,Yang Li,Yijie Yang,Xingrong Ju,Rong He. The preparation and physiochemical characterization of rapeseed protein hydrolysatechitosan composite films[J]. Food Chemistry. 2019

5. Suhaili Shamsi,Nurhuda Elias,Seri Narti Edayu Sarchio,Faizah Md Yasin. Gallic Acid Loaded Graphene Oxide Based Nanoformulation (GAGO) as Potential Anti-bacterial Agent against Staphylococcus aureus[J]. Materials Today: Proceedings. 2018

6. Sunil Meti,Mohammad R. Rahman,Md. Imteyaz Ahmad,K. Udaya Bhat. Chemical free synthesis of graphene oxide in the preparation of reduced graphene oxide-zinc oxide nanocomposite with improved photocatalytic properties[J]. Applied Surface Science . 2018

7. Zhang Jiaqi,Li Jin,Liu Xiangyu. Ternary nanocomposite ZnO-g-C3N4-Go for enhanced photocatalytic degradation of $\mathrm{RhB}[\mathrm{J}]$. Optical Materials,2021,119: 